\title{
Is the sugar intestinal permeability test a reliable investigation for coeliac disease screening?
}

\author{
C Catassi, E Fabiani, I M Rätsch, A Bonucci, M Dotti, G V Coppa, P L Giorgi
}

\begin{abstract}
Background-The lactulose/mannitol (L/M) intestinal permeability test is a simple, non-invasive screening test for coeliac disease. The reliability of the $L / M$ test has so far only been tested in selected groups of patients with coeliac disease.

Aim-To evaluate the reliability of the LM test in a group of patients with coeliac disease who had been diagnosed during mass serological screening of the general population.
\end{abstract}

Patients and methods-Twenty nine patients with coeliac disease detected by screening and 54 age matched coeliac disease free controls aged 11-15 years underwent an $L / M$ test with $5 \mathrm{~g}$ lactulose and $\mathbf{2} \mathbf{g}$ mannitol in isotonic aqueous solution. Urinary sugars were measured by high performance liquid chromatography.

Results-The median \% urinary recovery of lactulose (lactulose UR) was significantly higher in patients with coeliac disease than in controls $(0.63 v 0.18$, p<0.001). The mean mannitol $\% \mathrm{UR}$ was lower in patients with coeliac disease than in controls $(17.6 v 18.5)$ but the difference was not significant. The median urinary $\mathrm{L} \% / \mathrm{M} \%$ ratio was significantly higher in patients with coeliac disease than in controls $(0.038 v 0.014, p<0.001)$. However, 16 of the 29 patients with coeliac disease showed an $L \% / M \%$ ratio within normal limits $(<0 \cdot 044)$.

Conclusions-The L/M intestinal permeability test is not a valuable tool for screening of coeliac disease in the general population. The pattern of the urinary probe recovery suggests that many patients with coeliac disease could remain symptomless because the extent of their intestinal mucosal damage is small ("short" coeliac disease).

(Gut 1997; 40: 215-217)

Department of Pediatrics, University of Ancona, Italy

C Catassi

E Fabiani

I M Rätsch

A Bonucci

G V Coppa

P L Giorgi

Department of

Pediatrics, Palazzolo Hospital (Brescia),

Italy

$M$ Dotti

Correspondence to: Dr C Catassi,

The lactulose/mannitol (L/M) intestinal permeability test is a simple investigative tool currently used in the diagnostic investigation of patients with suspected coeliac disease. After an orally administered dose of sugar probes, patients with active coeliac disease usually show higher lactulose and lower mannitol urinary recovery (UR), with a pronounced increase in the L/M UR. These changes have mostly been found in selected groups of symptomatic patients. ${ }^{12}$
In recent years it has become clear that many patients with coeliac disease escape diagnosis. ${ }^{3}$ Despite typical coeliac damage at jejunal biopsy, many patients often show only minor symptoms or are apparently healthy. Treatment of these patients with a gluten-free diet is nevertheless recommended to prevent long term complications of coeliac disease, such as infertility or malignancy. ${ }^{4}$ Many patients with coeliac disease can be identified through serological screening of the general population using markers of coeliac disease such as the antigliadin antibodies (AGAs) as the screening test. $^{5}$ The reliability of the sugar intestinal permeability test as a screening tool for undiagnosed coeliac disease has not yet been determined.

We recently screened a general population sample of teenagers for coeliac disease by performing a serum IgG and IgA-AGA assay as the first level screening test. ${ }^{5}$ Most of the patients with coeliac disease detected by screening also had an L/M test at the diagnosis. The results of the intestinal permeability study on 29 coeliac patients detected by screening and on 54 age matched controls without coeliac disease are reported here.

\section{Patients and methods}

Both patients and controls took part in a coeliac disease screening project, the design of which has previously been published. ${ }^{5}$ Briefly, 7293 teenagers attending compulsory secondary school were screened using the serum IgG-AGA test and the IgA-AGA test, with the informed consent of their parents. Positive cases underwent a second step evaluation including a repeat of the AGA test, the IgA class antiendomysium antibody (AEA) test, and serum immunoglobulin measurement followed by a jejunal biopsy in those who showed at least one of the following: $(a)$ a positive IgA-AGA test, (b) a positive AEA test, (c) a positive IgG-AGA test and selective IgA deficiency. This diagnostic algorithm allowed the detection of 38 patients with coeliac disease in this population sample.

The intestinal permeability study was performed on 29 of the 38 patients with coeliac disease detected by screening. The other nine patients did not undergo the L/M test because of contingent problems, such as no fasting or incomplete sugar ingestion. There were 11 male and 18 female patients, with a mean (SD) age of $12.9(1.0)$ (range $11.6-14 \cdot 1$ ) years. In each patient the diagnosis of coeliac disease was 
confirmed by intestinal biopsy according to currently accepted criteria. ${ }^{6}$ All the patients with coeliac disease in this study were either free from major symptoms or showed a low grade intensity illness. The most common clinical findings were iron deficiency, recurrent abdominal pain, and behavioural disturbances.

\section{CONTROLS}

These were 54 randomly selected age matched students participating in the coeliac screening. They were considered to be free of coeliac disease because of normal results in the IgGAGA test, the IgA-AGA test, the AEA test, and the serum immunoglobulin measurements. In a large multicentre study the negative predictive value of this serological pattern was as high as $99 \cdot 3 \% .^{7}$ Controls comprised 23 male and 31 female subjects with a mean age of 12.7 (SD 0.9) (range $11 \cdot 3-14 \cdot 0$ ) years.

\section{METHODS}

The L/M test was performed after an overnight fast and bladder emptying. A total of $5 \mathrm{~g}$ lactulose and $2 \mathrm{~g}$ mannitol in $150 \mathrm{ml}$ water was given orally (isotonic load). All urine passed in the subsequent five hours was collected, the volume recorded, and an aliquot preserved at $-20^{\circ} \mathrm{C}$ with sodium azide. The urinary concentration of each sugar was measured by high performance liquid chromatography as previously described ${ }^{8}$ and the ratio of recovered to ingested sugar was calculated as a percentage (lactulose $\%$ UR and mannitol\% UR). The ratio of the lactulose $\%$ UR to the mannitol\% UR ( $\mathrm{L} \% / \mathrm{M} \%)$ was evaluated.

Intestinal biopsy specimens were taken at the Traitz ligament by a Watson capsule under fluoroscopic control.

STATISTICAL ANALYSIS

Whereas the mannitol\% UR values showed a normal distribution, the lactulose $\%$ UR and the $\mathrm{L} \% / \mathrm{M} \%$ distributions were positively skewed. A normal plot showed that both the lactulose $\%$ UR and the $\mathrm{L} \% / \mathrm{M} \%$ values fitted a Gaussian distribution after a square root $(V)$

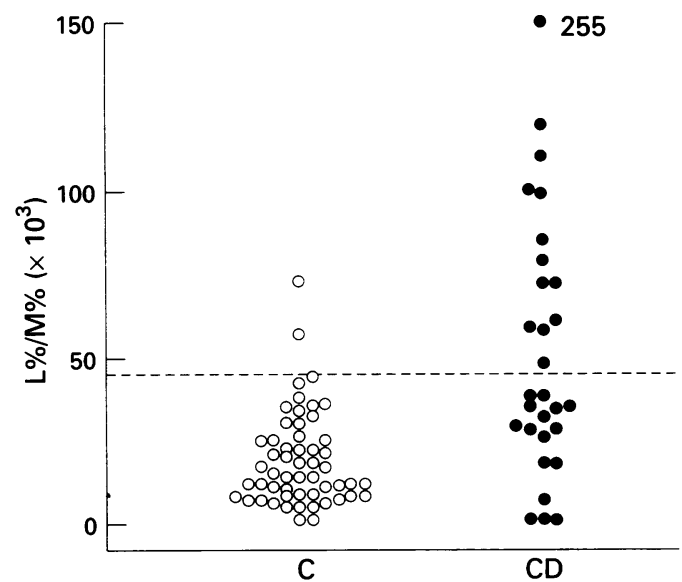

Figure 1: $L \% / M \%$ urinary ratio $\left(\times 10^{3}\right)$ in patients with coeliac disease (CD) and in the control (C) group. The dotted line indicates the upper limit of normal (0.044). transformation. The results of the $\mathrm{L} / \mathrm{M}$ test in control subjects were used to fix the normal limits of the permeability indexes (one tail $95 \%$ tolerance interval). The cut off values were established as follows: (a) mean $+1.65 \mathrm{SD}$ of the $V$ lactulose $\%$ UR; $(b)$ mean -1.65 SD of mannitol\% UR; $(c)$ mean +1.65 SD of the $V$ $\mathrm{L} \% / \mathrm{M} \%$. The results of the intestinal permeability study in controls and patients with coeliac disease were compared by either the Mann-Whitney $U$ test (median lactulose $\%$ UR and median $\mathrm{L} \% / \mathrm{M} \%$ ) or the Student's $t$ test for independent samples (mean mannitol\% UR).

The difference between the result of the haematological investigations in patients with coeliac disease with either a normal or a pathological value of the $\mathrm{L} \% / \mathrm{M} \%$ urinary ratio has been analysed by a Student's $t$ test for independent samples.

\section{Results}

Figures 1 and 2 show the results of the intestinal permeability study. The median lactulose $\%$ UR was significantly higher in patients with coeliac disease than in controls $(0.63 v 0.18$, $\mathrm{p}<0.001$ ); the mean mannitol\% UR was lower in patients with coeliac disease than in controls $(17.6 v 18 \cdot 5)$ but the difference was not significant. The median L\%/M\% UR was significantly higher in patients with coeliac disease than in controls $(0.038 v 0.014, \mathrm{p}<0.001)$. However, 16, 15, and 27 of the 29 patients with coeliac disease respectively showed an $\mathrm{L} \% / \mathrm{M} \%$ ratio, a lactulose $\% \mathrm{UR}$, and a mannitol\% UR within normal limits. Based on the $\mathrm{L} \% / \mathrm{M} \%$ UR, regarded as the most discriminating permeability index between controls and patients with coeliac disease, ${ }^{9}$ the sensitivity of the intestinal permeability test (subjects with a positive test $\times 100 /$ number of patients with coeliac disease) was $45 \%$.

The comparison between patients with coeliac disease with a normal $\mathrm{L} \% / \mathrm{M} \%$ urinary ratio and those with an $\mathrm{L} \% / \mathrm{M} \%$ UR higher than the cut off level $(0 \cdot 044)$ showed no significant difference in clinical presentation, the serological coeliac disease marker pattern, the histological picture, and the routine haematological investigations. The mean serum iron

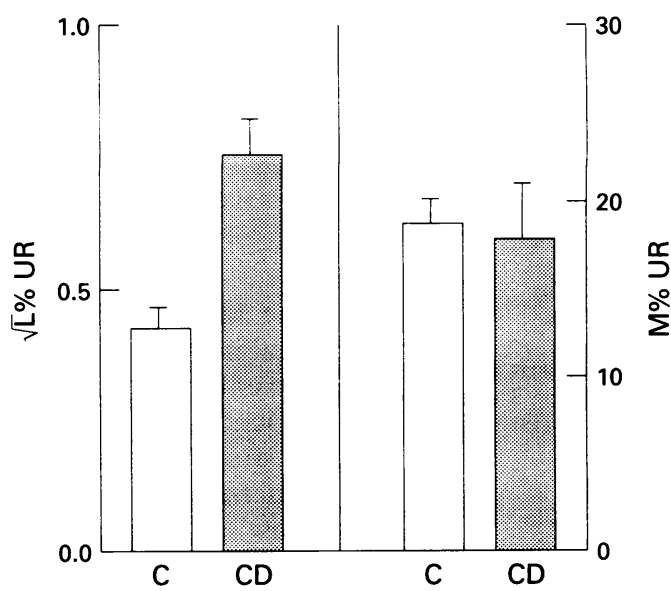

Figure 2: $\sqrt{ } L \%$ UR and $M \%$ UR in patients with coeliac disease (CD) and in controls (C). Data are expressed as mean (SEM). 
concentration was 51 (SD 17) $\mu \mathrm{g} / \mathrm{dl}$ in patients with coeliac disease with an abnormal $\mathrm{L} \% / \mathrm{M} \%$ UR and 71 (SD 31) in patients with coeliac disease with a normal permeability test, but the difference was not significant $(p=0 \cdot 09)$.

\section{Discussion}

A unique feature characterised the patients with coeliac disease investigated in this study; they were apparently healthy students who had been diagnosed through a mass serological screening. Although a typical gluten sensitive enteropathy was found at the intestinal biopsy in all cases, most of these patients with coeliac disease were either clinically silent or reported only minimal complaints. Could these patients have been suspected of having the disease after less invasive tests (not requiring a blood sample to be taken) such as the sugar intestinal permeability test? This hypothesis seemed plausible, as previous studies had suggested that the sensitivity of the L/M test in diagnosing coeliac disease could be as high as $96-100 \% .^{1011}$

Patients with coeliac disease detected by screening tended to have abnormal results in the intestinal permeability test with monodisaccharides, as has been shown in patients referred to hospital. ${ }^{1}$ However, neither the $\mathrm{L} \% / \mathrm{M} \%$ UR nor the other permeability indexes provided clear discrimination between controls and patients with coeliac disease. This finding is at variance with our previous results showing that the sugar intestinal permeability test is a valuable screening investigation in children referred for clinically suspected coeliac disease. ${ }^{8}$ We would argue that the sugar intestinal permeability test cannot be considered as a useful tool for coeliac disease screening on the general population as high sensitivity (a low number of false negative results) is a critical requirement for a first level screening test. ${ }^{12}$ This is especially true when the disease is not fully expressed on a clinical basis, as is often the case in coeliac disease.

It remains to be seen whether better diagnostic discrimination between patients with coeliac disease detected by screening and control subjects might be achieved by giving the sugar probes in hyperosmolar rather than isotonic solution. It is well known that hyperosmolar stress (test solution $1500 \mathrm{mmol} / \mathrm{l}$ ) increases the permeability of abnormal epithelia to the disaccharide probes. ${ }^{13}$ However, most workers have now abandoned the use of hyperosmolar stress in favour of the more physiological iso-osmolar tests. ${ }^{14}$

The median lactulose \% UR was significantly increased in our patients with coeliac disease detected by screening, as usually happens in patients with active coeliac disease. This is likely to be a consequence of their enteropathy, which was morphologically undistinguishable from that of clinically typical patients with coeliac disease. The mucosal damage might in turn lead to increased lactulose absorption by several mechanisms, such as the disruption of membrane integrity, changes in the tight junctions, and increased accessibility of the crypts to luminal contents. ${ }^{914}$ On the other hand, mannitol\% UR is usually reduced in patients with active coeliac disease. Although it is still unclear whether the main route of intestinal mannitol permeation is transcellular or paracellular, it is commonly assumed that reduced mannitol\% UR reflects the reduction in the total surface area of the small intestine caused by villous atrophy. ${ }^{15}$ In our patients with coeliac disease the mean mannitol UR was not significantly lower than in the control group. This might indicate that the absorptive surface was not grossly reduced in these patients. It can therefore be speculated that those detected by screening showed a clinically silent or mild form of coeliac disease because of a small extent of the mucosal damage ("short" coeliac disease). This hypothesis should be confirmed by the morphometric analysis of biopsy samples taken at different levels in the jejunum.

In conclusion, the results of this study indicate that the isotonic $\mathrm{L} / \mathrm{M}$ intestinal permeability test is not a valuable tool for coeliac disease screening of the general population. The pattern of the urinary recovery suggests that many patients with coeliac disease remain symptomless because they have limited damage to the mucosa of the small intestine.

1 Juby LD, Rothwell J, Axon ATR. Lactulose/mannitol test an ideal screen for celiac disease. Gastroenterology 1989 96: 79-85.

2 Hamilton I, Hill A, Bose B, Bouchier IAD, Forsyth JS Small intestinal permeability in pediatric clinical practice. F Pediatr Gastroenterol Nutr 1987; 6: 697-701.

3 Ferguson A, Arranz E, O'Mahony S. Clinical and pathological spectrum of coeliac disease - active, silent, latent, potential. Gut 1993; 34: 150-1.

4 Holmes GKT. Long-term health risks for unrecognized coeliac patients. In: Auricchio S, Visakorpi $\mathrm{JK}$, eds. Common food intolerances 1: epidemiology of coeliac disease. Basel: Karger, 1992: 105-18

5 Catassi C, Rätsch IM, Fabiani E, Rossini M, Bordicchia F Candela $\mathrm{F}$, et al. Coeliac disease in the year 2000 exploring the iceberg. Lancet 1994; 343: 200-3.

6 Working Group of the European Society of Paediatric Gastroenterology and Nutrition. Revised criteria for diag nosis of coeliac disease. Arch Dis Child 1990; 65: 909-11.

7 Bürgin-Wolff A, Gaze H, Hadziselimovic F, Huber $H$ Lentze MJ, Nusslé $D$, Reymond-Berther $C$ Antigliodin and antiendomysium antibody determination for coeliac disease. Arch Dis Child 1991; 66: 941-7.

8 Catassi C, Pierani P, Natalini G, Gabrielli O, Coppa GV Giorgi PL. Clinical application of a simple HPLC method for the sugar intestinal permeability test. $\mathcal{F}$ Pediatr Gastroenterol Nutr 1991; 12: 209-12.

9 Travis $S$, Menzies I. Intestinal permeability: functiona assessment and significance. Clin Sci 1992; 82: 471-88.

10 Bardella MT, Molteni N, Cesana B, Baldassarri AR Bianchi PA. IgA antigliadin antibodies, cellobiose/ mannitol sugar test and carotenemia in the diagnosis of and screening for celiac disease. Am $\mathcal{F}$ Gastroenterol 1991 ; 86: 309-11.

11 Vogelsang H, Genser D, Wyatt J, Lochs H, Ferenci $P$ Granditsch G, et al. Screening for celiac disease: a prospective study on the value of non-invasive tests. $\mathrm{Am}$ pective study on the value of
Gastroenterol $1995 ; 90: 394-8$.

12 Armitage P, Berry G. Statistical methods in medical research. Oxford: Blackwell Scientific Publications, 1994.

13 Weeler PG, Menzies IS, Creamer B. Effect of hyperosmolar stimuli and coeliac disease on the permeability of the human gastrointestinal tract. Clinical Sciences and Molecular Medicine 1978; 54: 495-501.

14 Bjarnason I, Macpherson A, Hollander D. Intestinal permeability: an overview. Gastroenterology 1995; 108: 1566-81

15 Dawson DJ, Lobley RW, Burrows PC, Notman JA, Mahon M, Holmes R. Changes in jejunal permeability and passive permeation of sugars in intestinal biopsies in coeliac disease and Crohn's disease. Clin Sci 1988; 74: 427-31. 